\title{
Ecosystem Services From Honey Beees Apis cerana Fabr. In Taman Hutan Raya (Tahura) Ir. H. Djuanda Dago Expert Bandung Ecology and Economically
}

\author{
Syayidah Nuriyah ${ }^{1}$, Arief Anshory Yusuf ${ }^{1,3}$, Wawan Hermawan ${ }^{1,2}$, Teguh Husodo ${ }^{1,2}$ \\ ${ }^{1}$ Postgraduate Program on Environmental Science, Padjadjaran University, Indonesia \\ ${ }^{2}$ Department of Biology, Faculty of Mathematics and Natural Sciences, Padjadjaran University, Indonesia. \\ ${ }^{3}$ Faculty of Economics, Padjadjaran University, Indonesia
}

\begin{abstract}
Pollination is one of the key components of global biodiversity and is very important for ecosystems in ensuring the maintenance of ecological processes, which are largely responsible for the successful reproduction of native plants. Apis cerana Fabr. including insect pollinators that are widely cultivated in the conservation forest Tahura Ir H Djuanda. Through survey and observation methods, it is known that some plants in Tahura are visited by honey bees, which have great potential for the effectiveness of plant pollination in Tahura. The types of plants visited by honey bees can be classified into agricultural and non-agricultural crops in a radius of 500 meters from Tahura. Based on the data, it is known that honey bees visited non-agricultural plants as many as 39 families and 83 species, while agricultural crops were 6 families and 17 species. So it can be concluded that bees prefer visiting non-agricultural crops rather than agricultural crops in Tahura, therefore the potential for pollination effectiveness by honey bees will be greater in non-agricultural crops such as plants in Tahura to maintain conservation forest biodiversity.
\end{abstract}

\section{Preliminary}

Humans benefit from the many resources and processes that natural ecosystems provide. Collectively, these benefits are known as ecosystem services. Ecosystem services are understood as conditions and processes that exist in natural ecosystems and species that enable them to sustain themselves and fulfill human life [4]; in other words, ecosystem services are a set of ecosystem functions that are useful for humans [8].

In another sense, ecosystem services are goods or services provided by ecosystems for human life $[3,10]$. For example, the amount of wood extracted from an ecosystem that depends at the request of the local community and the costs incurred in order to firewood can be obtained. The supply of ecosystem services will varying time, actual and potential supply in the future should be included in the valuation of ecosystem services [6].

According to [11], ecosystem services are divided into four categories, namely:

1. supply Services (provisioning): includes the results obtained from ecosystems, such as food (fruits, vegetables, grains), wood and fiber.

2. settings Services (regulating): eg climate arrangement, pollination (pollination), and control of the disease. settings Services including pest control and removal of carcasses
3. Cultural services (cultural) provide recreational services, inspiration for art and music, and spiritual value.

4. Support services (supporting), such as pollination, seed dispersal, purification of water and nutrient cycles, providing an important process for ecological communities and agricultural ecosystems

Tahura Djuanda is a conservation forest area with a high wealth of vegetation and various ecosystem services $[1,12]$. Among the forest ecosystem services provided by honey bees such as pollination services and honey production value.

Honey bees are pollinating insects that are widely cultivated by the people of Tahura Djuanda, namely the Apis cerana Fabr species. Apis cerana is a type of wild bee native to Asia. Current research on bees as honey producers or as pollinators on agricultural land $[7,9,13]$ has not assessed the value of bees in maintaining conservation forest ecosystems such as in Tahura. So a research was carried out with the aim of knowing the value of the ecosystem services of $A$. cerana honeybees from the bee forage plants and the value of honey production in Tahura Ir. H. Djuanda .

\section{Research methods}

The research method used is the method of roaming in the area within Tahura and around Tahura with a 
radius of 500 meters, structured interviews, and observing bee pollen being brought to the hive.

\section{Time and place}

The research was conducted for 3 months from January to March 2019 at Forest Park Raya (TAHURA) Ir H Djuanda Dago Pakar, Bandung.

\section{Tools and Materials}

Tool:

1. Insect net

2. Tweezers

3. Rapia

4. Camera

5. Test tube

6. Stirring Rod

7. object glass

8. Cover Glass

9. Binocular Microscope CX22

materials

1. Alcohol $70 \%$

2. $\mathrm{CH} 3 \mathrm{COOH}$ (vinegar)

\section{Data analysis}

Data analysis using SPSS and literature study from various literature.

\section{Results and Discussion}

\subsection{Location overview}

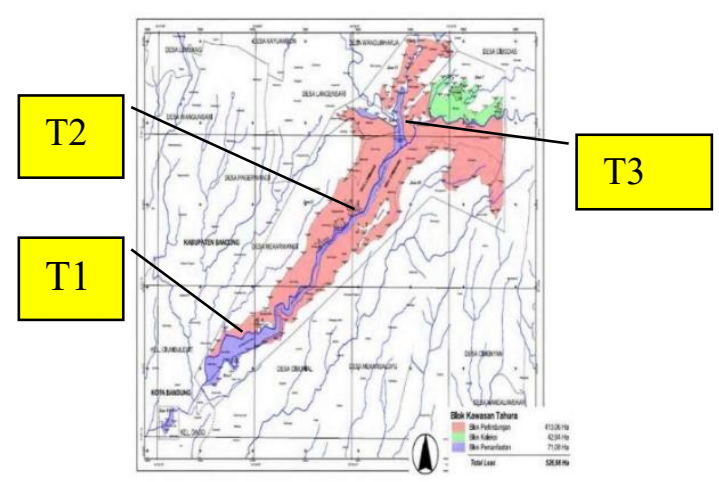

Fig.1. Maps of study at Tahura

Forest Park (TAHURA) Ir. H. Djuanda Dago Pakar was founded on January 14, 1985, geographically located $107^{\circ} 30^{\prime} 00$ "East Longitude and $6^{\circ} 52^{\prime} 00^{\prime \prime}$ South Latitude approximately 7 kilometers from the city center of Bandung (Fig.1).

Tahura Djuanda is an integrated conservation area between secondary nature and plantations with the type of Pinus (Pinus merkusii Jungh. \& Vriese), which is located in the Cikapundung River Basin and the Citarum Watershed which stretches from Curug Dago, Dago Pakar to Curug Maribaya which is part of the Gunung Pulosari forest group. The administration is in the territory of the District Ciburial Cimenyan Bandung District and parts of the village entrance Mekarwangi, Cibodas Village, Village Langensari and Wangunharja village, Lembang, West Bandung regency and village Dago [5]. In Fig. 2 , the research location shows three main points of honey bee maintenance, namely Batu Garok (T1 ), deer breeding (T2), and Maribaya (T3). The three of them are the center for $A$. cerana honey bee cultivation in Tahura, with the number of stups reaching more than 100 per location. The large number of hives indicates that the potential of Tahura as a provider of bee food is quite large, with a fairly high diversity of vegetation. It can be seen from IVI some vegetation in Tahura in Table $\mathbf{1}$, which also is a bee plant

Table 1. Importance Value Index (INP) based on the level of growth

\begin{tabular}{|c|c|c|c|c|}
\hline No & Habitus & Growth & Types of Plants & $\begin{array}{l}\text { INP } \\
(\%)\end{array}$ \\
\hline \multirow[t]{4}{*}{1} & Tree & Tree & Pinus (Pinus merkusii L) & 85.16 \\
\hline & & Pole & $\begin{array}{l}\text { Pulus Binong (Laportea } \\
\text { peltata) }\end{array}$ & 29.34 \\
\hline & & Stake & $\begin{array}{l}\text { Cinnamon ( Cinnamomum } \\
\text { burmannii } \mathrm{BL} \text { ) }\end{array}$ & 8.54 \\
\hline & & Seedling & $\begin{array}{l}\text { Ki Harendong } \\
\text { (Astronia spectabilis } \mathrm{Bl} \text { ) }\end{array}$ & 32.64 \\
\hline 2 & Shrubs & & $\begin{array}{l}\text { Kaliandra Merah } \\
\text { ( Calliandra } \\
\text { calothyrsus } \text { Meisn.) }\end{array}$ & 62.78 \\
\hline 3 & Herbs & & $\begin{array}{l}\text { Lempuyang Grass } \\
\text { ( Panicum repens } \mathrm{L} \text { ) }\end{array}$ & 35.24 \\
\hline 4 & Liana & & Rattan ( Calamus Sp. ) & 45.67 \\
\hline 5 & Epiphyte & & $\begin{array}{l}\text { Fur fern, monkey fern } \\
\text { ( Cibotium barometz (L.) } \\
\text { J.Sm). }\end{array}$ & 40.48 \\
\hline
\end{tabular}

Based on Table 1. The vegetation that has the highest IVI value based on the growth rate and habitus in Tahura Djuanda can be seen that those with the highest IVI are the dominant species, namely Pinus with an IVI value of $85.16 \%$ and calliandra with an IVI value of $62.78 \%$. Calliandra is the main food crop for honey bees.

In Table 2, it can be seen that at the research location a total of 83 types of forage plants were found in Tahura. Consists of 39 families were dominated by the family Fabaceae 10 species, Myrtaceae 8 species, Moraceae 5 species, Euphorbiaceae 4 species, Lauraceae 4 species, Anacardiaceae 3 species, Arecaceae 3 species, Malvaceae 3 species, Rutaceae 3 species, Zingiberaceae 3 species, Theaceae 1 species, Sapindaceae 2 species, Rosaceae 2 species, Pittosporaceae 2 species, Passifloraceae 2 species, Papilionaceae 2 species, and the rest only consists of 1 species, namely the family Annonaceae, Araliaceae, Bignoniaceae, Caesalpineaceae, Callophylaceae, Caricaceae, Dipterocarpaceae, Ebenaceae, Fagaceae, Gnetaceae, Lamiaceae, Lytheraceae, Magnoliaceae, Musaceae, Pineaceae, Rubiaceae, Salicaceae, Solanaceae, Sterculiaceae and Verbenaceae. 
Table 2. List of A.cerana honey bee forage plants in Tahura

\begin{tabular}{|c|c|c|c|c|}
\hline No. & Species name & Family & Region name & $\begin{array}{l}\text { Pollen/ } \\
\text { nectar }\end{array}$ \\
\hline 1 & Acacia mangingre Wild & Fabaceae & Acacia & $\mathrm{P} / \mathrm{N}$ \\
\hline 2 & $\begin{array}{l}\text { Adenovtherg povening (Teijsm } \\
\text { \& Binn) IC Nielsen }\end{array}$ & Fabaceae & Red Saga & $\mathrm{P}$ \\
\hline 3 & Albizia chinensis ( $(\mathrm{Qsb}$.) Merr. & Leguminosae & Sengon & $\mathrm{P}$ \\
\hline 4 & $\begin{array}{l}\text { Aleurites moivecang (L.) } \\
\text { Willd. }\end{array}$ & Euphorbiaceas & Candlenut & $P$ \\
\hline 5 & Amomon cardamomum Willd. & Zingiberaceae & cardamom & $\mathrm{N}$ \\
\hline 6 & Antidesma burius (L.) Spreng, & Euphorbiaceas & Buni / Huni & $\mathrm{N}$ \\
\hline 7 & $\begin{array}{l}\text { Archidendron iringa (Jack) } \\
\text { ICNielsen }\end{array}$ & Fabaceae & Jengkol & $\mathrm{P}$ \\
\hline 8 & $\begin{array}{l}\text { Arenga pinnata (Whorph) } \\
\text { Merr. }\end{array}$ & Arecacear & Aren & $\mathrm{P} / \mathrm{N}$ \\
\hline 9 & $\begin{array}{l}\text { Artocarpus } \\
\text { heterophyllus Lam. }\end{array}$ & Meraceas & Jackfruit & $P$ \\
\hline 10 & $\begin{array}{l}\text { Bauhinia purpurea DC-ex } \\
\text { Walp Blume }\end{array}$ & Fabaceae & butterfly leaves & N \\
\hline 11 & Bouea macrophylla Griff. & Anacardiaceae, & Gandaria & $\mathrm{P}$ \\
\hline 12 & Brugmansia candida Pers. & Solanaceae & Amethyst & $\mathrm{P} / \mathrm{N}$ \\
\hline 13 & $\begin{array}{l}\text { Calligudra } \\
\text { calothozsus Meissn. }\end{array}$ & Fabaceae & Red Calliandra & $\mathrm{P} / \mathrm{N}$ \\
\hline 14 & Calliandra tetragona. Meissn & Fabaceae & White Calliandra & $\mathrm{P}$ \\
\hline 15 & Calopholliom soulattri Burm of & Calophyllaceae & bintangur/ sulatri & $\mathrm{N}$ \\
\hline 16 & Camellia sinensis (L.) Kuntze & Theaceae, & Tea & $\mathrm{P} / \mathrm{N}$ \\
\hline 17 & $\begin{array}{l}\text { Cananga odorata (Lam.) } \\
\text { Hook. f. \& Thomson }\end{array}$ & Annonaceas & Ylang & $\mathrm{N}$ \\
\hline 18 & Caricapapava $\mathrm{L}$. & Caricaceae, & Papaya & $\mathrm{P}$ \\
\hline 19 & $\begin{array}{l}\text { Castamepsis argentea (Blume) } \\
\text { ADC }\end{array}$ & Fagaceae & Saninten & $P$ \\
\hline 20 & Cedrela mexicana,Roem & Meliaceae & Cedar Honduras & $\mathrm{P} / \mathrm{N}$ \\
\hline 21 & $\begin{array}{l}\text { Cinnamomum buromgonith (Dees } \\
\text { \& Th. Nees) }\end{array}$ & Lauracear & Cinnamon & $\mathrm{P} / \mathrm{N}$ \\
\hline 22 & $\begin{array}{l}\text { Cinnamomum } \\
\text { parthengyilon (Jack) Mejzn. }\end{array}$ & Lauraceae & lemongrass & $\mathrm{P} / \mathrm{N}$ \\
\hline 23 & $\begin{array}{l}\text { Cinnamomum } \\
\text { perzectum (Roxb.) Kosterm }\end{array}$ & Lauraceae. & Ki teja / selasihan & $\mathrm{P} / \mathrm{N}$ \\
\hline 24 & $\begin{array}{l}\text { Citrus moxima (Burm. F.) } \\
\text { Merr }\end{array}$ & Butaceaer & Pomelo & $\mathrm{P} / \mathrm{N}$ \\
\hline 25 & Citrus sinensis (L.) Osbeck & Rutaceaes & Local orange & $\mathrm{P} / \mathrm{N}$ \\
\hline 26 & Clidemia hirta (L.) D. Don & Melastomataceae, & Harendong Bulu & $\mathrm{P}$ \\
\hline 27 & $\begin{array}{l}\text { Coffea consephora } \text { Pierre ex } \\
\text { Froehner }\end{array}$ & Bubiaceare & Robusta coffee & $\mathrm{N}$ \\
\hline 28 & $\begin{array}{l}\text { Delenkx regia (Bojer ex } \\
\text { Hook.) }\end{array}$ & Fabaceae & Flamboyant & $\mathrm{N}$ \\
\hline 29 & Diospyros discolor Willd. & Ebenaceae, & Bisbul & $P$ \\
\hline 30 & Durie zibethinys Murray & Malvaceae & Durian & $\mathrm{P} / \mathrm{N}$ \\
\hline 31 & Dvsoxyizun excelsum Blume & Meliaceae & Monkey banana & $\mathrm{N}$ \\
\hline 32 & Elaeis guineensis Jacq. & Arecaceae & Palm oil & $\mathrm{N}$ \\
\hline 33 & $\begin{array}{l}\text { Enteralebiven } \\
\text { cyclacarpum (Jacq.) Griseb }\end{array}$ & Fabaceae & Sengon Bute & $\mathrm{P} / \mathrm{N}$ \\
\hline 34 & $\begin{array}{l}\text { Elxbetrag japonica } \\
\text { (Thunb) Lindl. }\end{array}$ & Rosaceae & Loquat & $\mathrm{N}$ \\
\hline 35 & Erythrina fiscenss & Papilionaceas & dadap, & $\mathrm{N}$ \\
\hline 36 & $\begin{array}{l}\text { Erythrina subumbrows (Hassk). } \\
\text { Merr. }\end{array}$ & Papilionacese & Dadap spare & N \\
\hline 37 & Eucalyptus citriodora Hook & Mrrtaceas & Lemon eucalyptus & $\mathrm{N}$ \\
\hline 38 & Eucalyptus deglupta Blume & Mrrtaceae & leda/ eucalyptus & $\mathrm{N}$ \\
\hline 39 & Eugenia cyminj (L.) Druce & Mrstaceae & Jamblang & $\mathrm{P} / \mathrm{N}$ \\
\hline 40 & Eugenia densilera (B1.) Mig. & Myctacear & $\begin{array}{c}\text { Jambu-jambuan } \\
\text { Ki sireum }\end{array}$ & $\mathrm{N}$ \\
\hline 41 & Euphorbia longan Lour, & Euphorbiaceae & Longan & $\mathrm{P} / \mathrm{N}$ \\
\hline 42 & $\begin{array}{l}\text { Eggraea fragrans Roxbeex } \\
\text { Carey \& Wall. }\end{array}$ & Rosaceae & strawberry & $\mathrm{P} / \mathrm{N}$ \\
\hline 43 & Ficus altissimg Blume & Moraceas & ficus tree & $P$ \\
\hline 44 & Ficus glomerata Blanco & Moraceas & Loa tree & $\mathrm{P}$ \\
\hline 45 & Ficus septica Burm. & Moraceas & Ki Ciat & $\mathrm{P}$ \\
\hline 46 & Ficus yorisgata, Blume & Moraceas & Ara Kondang & $\mathrm{P}$ \\
\hline 47 & $\begin{array}{l}\text { Eilicium decipiens (Weight \& } \\
\text { Am) }\end{array}$ & Sapindaceas & Kiara Payung & $\mathrm{N}$ \\
\hline 48 & $\begin{array}{l}\text { Eluceustia oukom Zoll. \& } \\
\text { Moritzi }\end{array}$ & Salicaceae & rukem/lobbies & $\mathrm{P} / \mathrm{N}$ \\
\hline 49 & Globba pendula $\mathrm{R} o x b$ & Zingiberaceae & Spicy mouse deer & $\mathrm{P} / \mathrm{N}$ \\
\hline 50 & Gmelina arborea Roxb. & Lamiaceae, & White Teak & $\mathrm{P}$ \\
\hline 51 & Gnetum gneman L. & Gnetaceae & melinis & $\mathrm{P} / \mathrm{N}$ \\
\hline 52 & Hibiscus similis Blume & Malvaceae, & Warudoxong & $\mathrm{N}$ \\
\hline 53 & $\begin{array}{l}\text { Kigelig aethiopica (Aubrevex } \\
\text { Sillans) }\end{array}$ & Bignoniaceae & Sausage tree & $\mathrm{P} / \mathrm{N}$ \\
\hline 54 & $\begin{array}{l}\text { Lagerstroemia speciosa }(\mathrm{L} .) \\
\text { Pers. }\end{array}$ & Luthraceas & bungur & $\mathrm{P}$ \\
\hline 55 & $\begin{array}{l}\text { Leucaena leucocephala (Lam.) } \\
\text { De Wit. }\end{array}$ & Leguminosae & White leadtree & $\mathrm{P}$ \\
\hline 56 & Mangifer a foetida Lour. & Anacardiaceae & $\begin{array}{l}\text { Mango chopped / } \\
\text { Limus }\end{array}$ & $\mathrm{P} / \mathrm{N}$ \\
\hline 57 & Mangifera $\times$ odorata Griffith. & Anacardiaceae, & Mango pakel & $\mathrm{P} / \mathrm{N}$ \\
\hline 58 & Melastom a malabathricum L. & Melastomataceae & Harendong & $\mathrm{P}$ \\
\hline
\end{tabular}

\begin{tabular}{|c|c|c|c|c|}
\hline 59 & Michelia champaca L. & Magnoliaceae & Manglid/campakg & $\mathrm{N}$ \\
\hline 60 & Murrene pouiculata (M). Jack & Rutaceas & Kamurning & $\mathrm{N}$ \\
\hline 61 & Musa paradisiaca $\mathrm{L}$. & Musaceae & Banana & $\mathrm{N}$ \\
\hline 62 & Nephelium lappaceum L. & Sanindaceae & Rambutan & $\mathrm{P} / \mathrm{N}$ \\
\hline 63 & Passifiora ecdulis Sims & Passifloraceae & $\begin{array}{l}\text { Purple passion } \\
\text { fruit }\end{array}$ & $\mathrm{P} / \mathrm{N}$ \\
\hline 64 & Passiffora ligularis Juss. & Passifloraceae & $\begin{array}{l}\text { Yellow passion } \\
\text { fruit }\end{array}$ & $\mathrm{P} / \mathrm{N}$ \\
\hline 65 & Perseg americana Mill. & Lauraceae. & Avocado & $\mathrm{P} / \mathrm{N}$ \\
\hline 66 & $\begin{array}{l}\text { Pinus merbusit Jungh \& } \\
\text { Vriese }\end{array}$ & Pinaceae & Pine & $P$ \\
\hline 67 & $\begin{array}{l}\text { Pittosporum } \\
\text { ferruginerem Dryand. ex } \\
\text { WTAiton. }\end{array}$ & Pittosporaceae & Ki Honje & $P$ \\
\hline 68 & $\begin{array}{l}\text { Pittosporum } \\
\text { moluccamm (Lam.) Miq. }\end{array}$ & Pittosporaceae & Ki Honje & $\mathrm{P}$ \\
\hline 69 & Psidium guajova L & Murtaceas & Guava / klutuk & $P$ \\
\hline 70 & Ricimus communis $\mathrm{L}$. & Euphorbiaceas & Kaliki / jarak & $\mathrm{N}$ \\
\hline 71 & Salascg edulis Reinx, & Arecaceas & Salak & $\mathrm{N}$ \\
\hline 72 & $\begin{array}{l}\text { Schefflera scandens (Blume) } \\
\text { R.Vig. }\end{array}$ & Araliaceae & Bamegiling & $P$ \\
\hline 73 & Schimg wallichii (DC.) Korth. & Theaceae, & Puspa & $\mathrm{P}$ \\
\hline 74 & $\begin{array}{l}\text { Senna sioneg (Lam.) HS Irwin } \\
\text { \& Bameby }\end{array}$ & Fabaceae & Venus & $\mathrm{P} / \mathrm{N}$ \\
\hline 75 & Shorea leprosula Mio. & Dipterocarpaceae, & Copper Meranti & $P$ \\
\hline 76 & Sterculia urceolata $\mathrm{Sm}$. & Sterculiaceae, & Hantap / Faloak & $P$ \\
\hline 77 & $\begin{array}{l}\text { Syzygizon equetom (Burm,f) } \\
\text { Alston }\end{array}$ & Mextacear & Water apple & $\mathrm{P}$ \\
\hline 78 & $\begin{array}{l}\text { Syzjgizm majaccemse (L.) } \\
\text { Merr. \& LiMPernx }\end{array}$ & Mxtacear & Guava bel & $\mathrm{P}$ \\
\hline 79 & 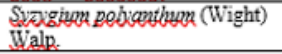 & Myxtacear & Regards & $\mathrm{P} / \mathrm{N}$ \\
\hline 80 & Tamarindus indica $\mathrm{L}$. & Caesalpiniaceae & Tamarind & $\mathrm{P} / \mathrm{N}$ \\
\hline 81 & Urena lobata L. & Malyaceae, & Pungeurutan & $\mathbf{P}$ \\
\hline 82 & Vitex pubescens. Vahl. & Verbenaceae & Laban wood & $\mathrm{P}$ \\
\hline 83 & Zingiber inflexum Blume. & Zingiberaceae & ginger ale & $\mathrm{N}$ \\
\hline
\end{tabular}

Fabaceae is the dominant bee food family in Tahura, such as acacia and butterfly flower plants which are a source of nectar and honey bee pollen. Acacia and butterfly flower plants belong to tree habitus, which is found in mixed forest habitats. Butterfly flower plants (Bauchinia purpurea) are widely planted in utilization blocks and areas near Japanese caves or Dutch caves along pedestrian tracks, this is because these plants have beautiful purple flowers that have aesthetic value for Tahura visitors.

Tabel 3. Forage crops of A. cerana honey bees on agricultural land

\begin{tabular}{|c|c|c|c|c|}
\hline No. & Latin name & Family & Region name & $\begin{array}{l}\text { Pollen / } \\
\text { nectar }\end{array}$ \\
\hline 1 & Arachis hxpogaea L. & Fabaceae & Peanuts & $\mathrm{P} / \mathrm{N}$ \\
\hline 2 & $\begin{array}{l}\text { Brassica juncea (L.) } \\
\text { Czenn. }\end{array}$ & Brassicaceae & $\begin{array}{l}\text { Mustard } \\
\text { greens }\end{array}$ & $\mathrm{P} / \mathrm{N}$ \\
\hline 3 & $\begin{array}{l}\text { Brassica } \\
\text { oleracea I. var. italica }\end{array}$ & Brassicaceae & Broccoli & $\mathrm{P}$ \\
\hline 4 & $\begin{array}{l}\text { Brassica oleracea } \mathrm{L} \text {. } \\
\text { var. capitata }\end{array}$ & Brassicaceae & Cabbage & $\mathrm{P}$ \\
\hline 5 & Capsicum annuum $\mathrm{L}$. & Solanaceae & Great chili & $\mathrm{P} / \mathrm{N}$ \\
\hline 6 & Capsicum frutescens $\mathrm{L}$. & Solanaceae & $\begin{array}{l}\text { Cayenne } \\
\text { pepper }\end{array}$ & $\mathrm{N}$ \\
\hline 7 & Cucumis sativus L. & Cucurbitaceae & Cucumber & $\mathrm{P} / \mathrm{N}$ \\
\hline 8 & $\begin{array}{l}\text { Fragaria } \times \\
\text { gnanassa (Weston) } \\
\text { Duchesne }\end{array}$ & Rosaceae & Strawberry & $\mathrm{P} / \mathrm{N}$ \\
\hline 9 & Glycine max (L.) Merril & Fabaceae & Soy & $\mathrm{P} / \mathrm{N}$ \\
\hline 10 & Oryza sativa L. & Poaceas & rice & $\mathrm{P}$ \\
\hline 11 & Phaseolus vulgaris $\mathrm{L}$. & Fabaceae & Bean & $\mathrm{P} / \mathrm{N}$ \\
\hline 12 & $\begin{array}{l}\text { Sechium edule (Jacq.) } \\
\text { Sw. }\end{array}$ & Cucurbitaceae & Chayote & $\mathrm{N}$ \\
\hline 13 & $\begin{array}{l}\text { Solanum } \\
\text { lycopersicum } \mathrm{L} \text {. }\end{array}$ & Solanaceae & Tomato & $P$ \\
\hline 14 & Solanum melongena $\mathrm{L}$. & Solanaceae & $\begin{array}{l}\text { Purple } \\
\text { eggplant }\end{array}$ & $\mathrm{P} / \mathrm{N}$ \\
\hline 15 & Solanum tuberosum L. & Solanaceae & Potato & $\mathrm{P} / \mathrm{N}$ \\
\hline 16 & Zea mays L. & Poaceas & corn & $\mathrm{P}$ \\
\hline 17 & $\begin{array}{l}\text { Vigna unguiculata (L.) } \\
\text { Walp. }\end{array}$ & Fabaceae & long beans & $\mathrm{P} / \mathrm{N}$ \\
\hline
\end{tabular}

Based on the observations in Table 3, 6 families and 17 species of bee food plants were obtained, consisting of Fabaceae, Brassicaceae, Solanaceae, Cucurbitaceae, Rosaceae, and Poaceae. 
Various studies have shown that pollination services by insects are higher on agricultural land bordering forests and other semi-natural lands compared to land bordering other agricultural lands. [2] found that there was a negative relationship between the diversity and abundance of pollinating insects and the distance from the forest boundary, the farther from the forest the diversity and abundance of pollinating insects was getting smaller.

\subsection{Observation of Pollen loads carried by honey bees to the hive}

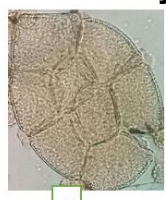

a

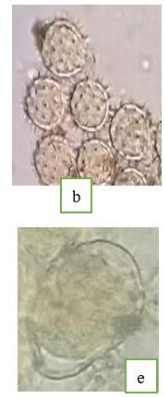

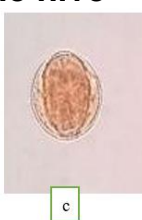

Fig. 3. Pollen loads are dominant in honey bees. Fabaceae (a), Asteracea b), Euphorbiaceas (c), Leguminoce (d), and pineaceae (e,). In totan
Fig. 2. Shows some images of the dominant pollen loads of A. cerana honey bees in Tahura. Total pollen lo ads of honeybees brought ke nests based observations laborato rium there are 45 species of plants. Then do the grouping po 1 len loads dominant, the pollen of a rare species, and pollen from tana man with high IVI Tahura. Dominant pollen loads carried by bees: Asteraceae 4513 eggs per head. Pollen loads: Rare plant species based on IUCN: Dipterocarpaceae, namely Shorea leprosula (copper meranti). Pollen loads with high INP: Fabaceae and Pineaceae

\subsection{The economic value of honey bees}

Table 3. Annual productivity of honey bee cultivation in Tahura

\begin{tabular}{|c|c|c|c|c|c|}
\hline No. & Location & $\begin{array}{l}\text { The number of } \\
\text { filled stacks in } \\
\text { Tahura }\end{array}$ & $\begin{array}{l}\text { Honey } \\
\text { produced }(\mathrm{kg})\end{array}$ & $\begin{array}{l}\text { The nominal } \\
\text { earned by farmer } \\
\text { groups per year } \\
\text { (Rp) }\end{array}$ & $\begin{array}{l}\text { Production value } \\
\text { for the manufacture } \\
\text { and maintenance of } \\
\text { bee stew per year }\end{array}$ \\
\hline 1 & Cave Belanda 1 & 20 & 40 & 10.000 .000 & 500.000 \\
\hline 2 & Cave Belanda 2 & 16 & 34 & 8.500 .000 & 400.000 \\
\hline 3 & Pos 2 & 11 & 20 & 5.000 .000 & 275.000 \\
\hline 4 & Maribaxa & 50 & 150 & 37.500 .000 & 1.250 .000 \\
\hline 5 & PLN 1 & 10 & 25 & 6.250 .000 & 250.000 \\
\hline 6 & PLN 2 & 15 & 45 & 11.250 .000 & 375.000 \\
\hline & PLN 3 & 7 & 21 & 5.250 .000 & 175.000 \\
\hline 8 & PLN 4 & 10 & 22 & 5.500 .000 & 250.000 \\
\hline 9 & PLN 5 & 12 & 27 & 6.750 .000 & 300.000 \\
\hline 10 & Deer Breeding tracks 1 & 16 & 38 & 9.500 .000 & 400.000 \\
\hline 11 & Deer Breeding tracks 2 & 12 & 28 & 7.000 .000 & 300.000 \\
\hline 12 & Deer Breeding tracks 3 & 11 & 22 & 5.500 .000 & 275.000 \\
\hline 13 & Deer Breeding 1 & 14 & 30 & 7.500 .000 & 350.000 \\
\hline 14 & Deer Breeding 2 & 18 & 40 & 10.000 .000 & 450.000 \\
\hline 15 & Deer Breeding 3 & 27 & 56 & 14.000 .000 & 675.000 \\
\hline 16 & Deer Breeding 4 & 16 & 33 & 8.250 .000 & 400.000 \\
\hline 17 & Deer Breeding 5 & 18 & 37 & 9.250 .000 & 450.000 \\
\hline 18 & Deer Breeding 6 & 17 & 36 & 9.000 .000 & 425.000 \\
\hline 19 & Danouxangan Valley & 20 & 42 & 10.500 .000 & 500.000 \\
\hline 20 & Garak Stone & 48 & 120 & 30.000 .000 & 1.200 .000 \\
\hline & Total & 368 & 866 & 216.500 .000 & 9.200 .000 \\
\hline & Average/years & & & 18.041 .666 & 766.666 \\
\hline
\end{tabular}

The honey bee productivity value in producing honey in Tahura which is the gross benefit value obtained is Rp.216.500.000 / year, with an average of Rp. 18.041.666 / farmer / year.

Then the production value of the bee hive is $\mathrm{Rp}$. 9.200.000/year, with an average of Rp.766,666/ farmer/year.

The average age of beekeeping per hive is 2 years. The price of 1 bee stup is around Rp.
100.000 Then the cost per farmer/year is Rp. 50,000. In total there are about 368 hives in Tahura, then the value of the outlay for maintaining the hive is:

- 368x IDR 50,000 = 18.400.000

Then the total net production value of honey or the direct benefit value (DUV) of honey bees in Tahura:

DUV $=\quad$ Rp. $216.500 .000-\quad$ Rp. 9.200 .000

Rp. $18.400 .000=$ Rp. $188.900 .000 /$ year

Then the value of the direct benefits of honey bees in Tahura is Rp. 188.900.000/year.

\section{Conclusion}

- Honey bees A.cerana provide various types of ecosystem services in Tahura, including pollination services and honey production. The ecological services that honey bees provide include pollination. The existence of honey bees has the potential for conservation of Tahura vegetation through pollination of dominant plants and rare plants seen from bee pollen collection. Bees are known to take plant pollen of 39 families and 83 species from vegetation in Tahura, and 6 families 17 species from agricultural crops $500 \mathrm{~m}$ radius from Tahura.

- In addition, the honey bee A.cerana provide economic services through the production of honey valued at 216.500.000 / year from 20 beekeepers. And the value of direct benefits (DUV) of Rp. 188.900.000/year

- So it can be concluded that bees provide ecosystem services ecologically and economically to the sustainability of the Tahura Ir. H. Djuanda ecosystems.

\section{References}

1. Arief, A. M, Pengembangan Aktivitas Wisata di Taman Hutan Raya Ir. H. Djuanda Bandung Jawa Barat, Jurnal Sorot Vol 8 No 2: 1 - 190 (2013)

2. Bailey, S., Requier, F., Nusillard, B., Roberts, S. P. M., Bouget, C., Potts, S. G., Distance from forest edge affects bee pollinators in oilseed rape fields. Ecology and Evolution 2014; 4(4): 370-380 (2014)

3. Costanza, R., Cumberland, J., Daly, H., Goodland, R., dan Norgard, R., An Introduction to Ecological Economics. St. Lucie Press, Boca Raton, Florida (1997)

4. Daily, G. C., Nature's Services: Societal Dependence on Natural Ecosystems. Island Press: Washington, DC (1997) 
5. Dinas Kehutanan Pemerintah Provinsi Jawa Barat, Booklet Taman Hutan Raya Ir. H. Djuanda. Dinas Kehutanan (2015)

6. Drepper, F.R. and B.Å. Månsson, Intertemporal valuation in an unpredictable environment. Ecological Economics 7: 43-67 (1993)

7. Kasno, Hasan, A. E., Efendi, D. S., Syaefudin., Efektivitas 3 Spesies Lebah Madu sebagai Agen Polinasi untuk Meningkatkan Produktivitas 40\% Biji Jarak Pagar Jatropha curcas pada ekosistem Iklim Basah. Jurnal Ilmu Pertanian Vol.15: 25-3 (2010)

8. Kremen, C., Managing Ecosystem Services: What Do We Need to Know About Their Ecology? Journal Ecology Letters, 8: 468-479 (2005)

9. Masfufah, I., Keanekaragaman Serangga Penyerbuk dan Efektivitasnya dalam Pembentukan Buah Pala Myristica fragrans Houtt: Myristicaceae. Tesis. IPB: Bogor (2010)

10. Millenium Ecosystem Assessment (MEA), Ecosystems and Human Well-being: A Framework for Assessment. Island Press: Washington, D.C. (2003)

11. Millenium Ecosystem Assessment (MEA), Ecosystems and Human Well-being: Synthesis. Island Press: Washington, D.C (2005)

12. Purwanto, A. B., Valuasi Ekonomi Wana Wisata Taman Hutan Raya Juanda dengan menggunakan Pendekatan "Travel Cost Method". Tesis Teknik Lingkungan Pascasarjana Institut Teknologi Bandung (1998)

13. Widhiono, I., Sudiana, E., dan Sucianto, E. T. Potensi Lebah Lokal dalam Peningkatan Produksi Buah Strawberry Fragaria x ananassa. Jurnal inovasi Vol 6 no 2: 163-168 (2012) 